\title{
Energy consumption for Video Transmission in Mobile Ad Hoc Networks
}

\author{
Arun Kumar \\ SC\&SS \\ Jawaharlal Nehru University \\ New Delhi, India-110067
}

\author{
D. K Lobiyal \\ SC\&SS \\ Jawaharlal Nehru University \\ New Delhi, India-110067
}

\begin{abstract}
In the mobile ad hoc network the energy consumed by the transmitter and receiver is more significant due the more energy consumption in the transmitting and receiving of the data. Therefore energy consumption in the devices is needed to optimize. In this paper we analysis the Doppler shift effect on the energy consumption of the transmitter in video transmission over the mobile ad hoc network. In this work we analyses the energy consumption of the transmitter device over the MQAM modulation and vary the average velocity of the devices.
\end{abstract}

\section{Keywords}

Mobile Ad Hoc Network, MQAM, Doppler Effect, Energy Consumption,

\section{INTRODUCTION}

In recent development of communication, we have seen that wireless multimedia is growing rapidly. Due to this, need for number of mobile devices is also increasing. These mobile devices operate on the battery power. The battery power consumption affects the network connectivity and thus network life which have impact on video quality. Therefore, battery power consumption by these devices is of great concern to researchers due to the limited power supply [1, 2,3, 4,5]. Therefore, the minimization of battery power consumption is a key research area to enhance battery life and network life as well.

Video transmission in mobile ad hoc network has two major challenges for maintaining the minimum quality of service (QoS) requirements. One is to maintain the real time operation of video data and the other is minimizing power consumption by the mobile devices.There are many recent researches going on the energy saving like energy efficient routing [2, 3, 6, 7], energy aware scheduling of video, power consumption of the circuit of transmitter and receiver [1, 2,7, 8], etc. The mobile device comprises radio modem and data interface which are required for the communication $[2,6,9,10]$. During video transmission in mobile ad hoc network approximately $75 \%$ energy is consumed by the radio modem. Therefore, energy consumption by transmitter and receiver circuit is intensely considered by the researchers. In IEEE 208.11 b wireless LAN card based on Intresil's PRISM II chipset, power amplifier (PA) consumes $600 \mathrm{~mW}, 110 \mathrm{~mW}$ by MAC processor, digital baseband consumes $170 \mathrm{~mW}$ and $240 \mathrm{~mW}$ consumed by the analog circuits $[1,2,7,9]$.

\section{RELATED WORK}

Energy minimization is important goal for the video transmission in mobile ad hoc network. Due to the limited power of battery of the mobile node in the ad hoc network path failure occur. The path failure cause video distortion which affect the video quality [4,5,11].In video communication over mobile network, power consumption is of great concern. Significant power is consumed in transmission and receiving video packet by the mobile device circuits. In this most of the battery power consumed by the RF circuit. In the RF front, energy consumption model [1, 2, 3, 7, 9] consider physical layer parameters bit rate, bit error rate (BER), bandwidth, modulation, for optimization of the energy consumption in the transmitter and receiver circuit (RF). The other non-physical layer parameters like: thermal noise, fading, harmonic distortion also affect the energy consumption in the wireless communication $[2,3,7]$. In thisenergy efficient video transmission schemesare developed that considersthe frame by frame, group of pictures (GOP)-GOP transmission and buffer related energy consumption. The client-buffer-related energy efficient video transmission (CBEVT) and smoothing strategy schemes reduce the power consumption in the video transmission. While CBEVT algorithm reduces energy consumption by sooner transmitting video data with the peak bit rate which frequently exceeds the transmission rate. This corresponds to the energy minimization at the modulation level. Smoothing algorithms optimize the energy consumption when buffer is large and average bit rate of video whichare close tothe energy minimization transmission rate [7].

The minimization of transmitter and receiver power for the video transmission requires minimizing the RF front-end power consumption. The standard transmitter and receiver block diagram is shown in figure1. It consists of digital to analog convertor, power amplifier, mixer, and RF synthesizer, different type of filters, noise amplifier and analog to digital convertor.

In this work we compute the power consumption of transceiver for the transmission of video packet. There are noise and other effects which have major impact on the power consumption. These effects are 1/f noise, harmonic distortion and Doppler Effect. 1/f noise occurs due the thermal noise, frequency dependency, and other factors. Harmonic distortion is caused by nonlinearity of the system.

Doppler shift is defined by the change or shift in the frequency due to the mobility of the transmitter and receiver devices in mobile ad hoc network [12]. If they are moving toward each other then frequency will increase and if they move far away from each other, then the frequency will be lower. Doppler shift $f_{d}$ is given as

$$
f_{d}=\frac{v}{\lambda}
$$




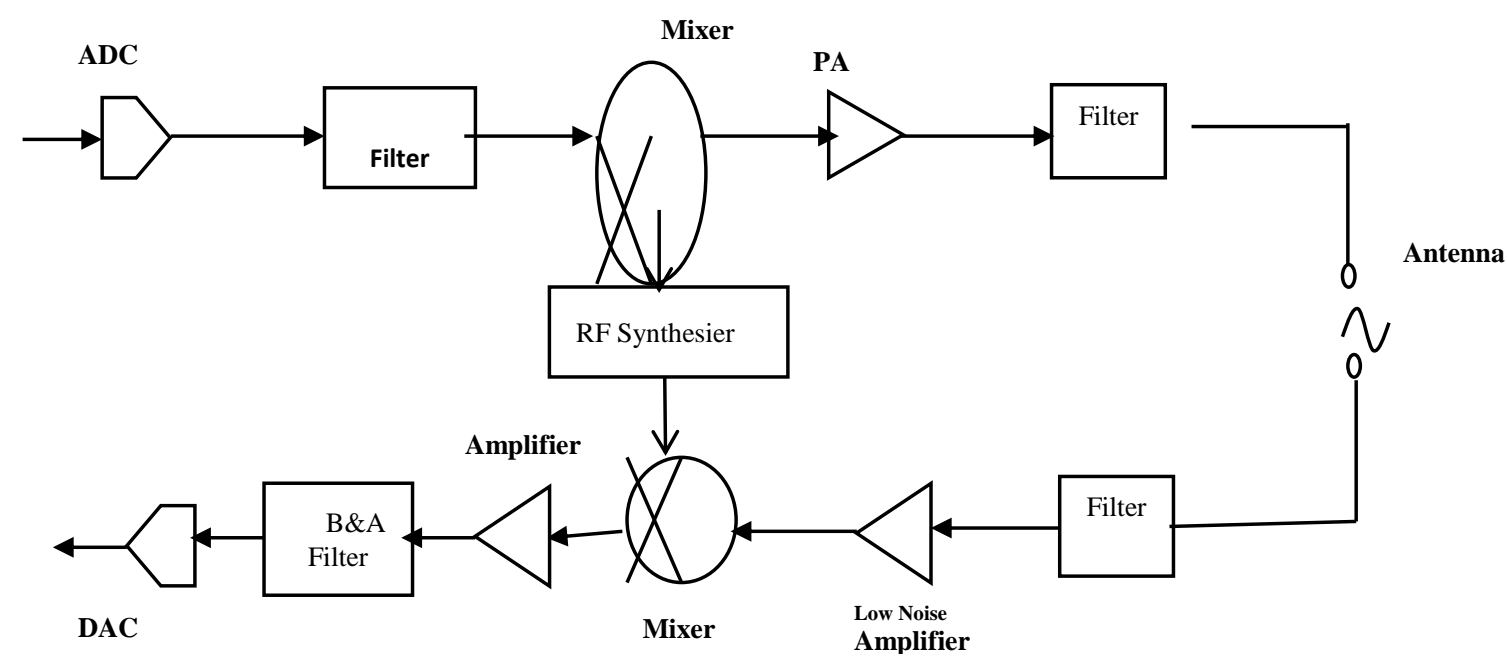

Fig1: Block diagram of transmitter and receiver system

\section{PROPOSED WORK}

Where $v$ is the average velocity of sender mobile devices and $\lambda$ is the wavelength of the signal.

From the papers $[7,9]$ the ratio of the signal peak power to its rms value PAR is defined as $\operatorname{PAR}(d B)=10 \log _{10}\left(P_{\text {peak }} / P_{\text {rms }}\right)$. Where $\mathrm{P}_{\text {peak }}$ represent peak power and $\mathrm{P}_{\mathrm{rms}}$ is average power. For the QAM modulation at the modulation level b, PAR is defined as

$$
\begin{gathered}
P A R_{Q A M}=P A R_{\text {modulation }}(d B)+P A R_{\text {roll-off }}(d B) \\
P A R_{Q A M}=\log \left(\sqrt{\frac{3 \cdot\left(2^{b / 2}-1\right)}{2^{b / 2}+1}} \cdot P A R_{c}\right)+P A R_{\text {roll-off }}(d B)
\end{gathered}
$$

Where $\mathrm{PAR}_{\mathrm{c}}$ is the PAR of the carrier.

For the PSK modulation at modulation level b, PAR is defines as

$$
P A R_{Q A M}=\log \left(P A R_{c}\right)+P A R_{\text {roll-off }}(d B)
$$

Power amplifier (PA) is the most power consuming part in the transmitter. For the different modulation power consumption in PA is describe as follows [2, 7, 9]

For the MQAM modulation

$$
\begin{gathered}
P_{P A(M Q A M)}=\frac{P_{r m s}}{R} \cdot P A R \\
P_{P A(M Q A M)}= \\
\frac{16 \pi^{2} \cdot d^{2} \cdot L}{3 G_{r} G_{t} \lambda^{2} \cdot K}\left(2^{b}-1\right) \cdot N \cdot\left(Q^{-1}\left(\frac{1}{4}\left(1-\frac{1}{2^{b / 2}}\right)^{-1} b \cdot B E R\right)^{2}\right) P A R_{Q A M}
\end{gathered}
$$

For the MPSK modulation

$$
P_{P A(M P S K)}=\frac{4 \pi^{2} \cdot d^{2} \cdot L}{G_{r} G_{t} \lambda^{2} \cdot K} \cdot N \cdot\left(Q^{-1}\left(\frac{b}{2 \sin \left(\frac{\pi}{2^{b+1}}\right)} \cdot B E R\right)^{2}\right) P A R_{P S K}
$$

For the fading channel and Doppler Effect the power consumption will be changed therefore power is given as

$$
P_{\text {detected }}=\frac{P_{r m s} \cdot|h|^{2} \cdot G_{r} G_{t} \lambda^{\prime 2}}{16 \pi^{2} \cdot d^{2} \cdot L}
$$

, $h$ is fading factor

Power consumption in PA for the fading channel and Doppler Effect in MQAM modulation

$$
\begin{gathered}
P_{P A(M Q A M)}=\frac{16 \pi^{2} \cdot d^{2} \cdot L}{3 G_{r} G_{t} \lambda^{\prime 2} \cdot|h|^{2} \cdot K}\left(2^{b}-1\right) \cdot N \cdot\left(Q ^ { - 1 } \left(\frac{1}{4}(1-\right.\right. \\
\left.\left.\left.\frac{1}{2^{b / 2}}\right)^{-1} b \cdot B E R\right)^{2}\right) P A R_{Q A M}
\end{gathered}
$$

Power consumption in PA for the fading channel and Doppler Effect in MPSK modulation

$$
P_{P A(M P S K)}=\frac{4 \pi^{2} \cdot d^{2} \cdot L}{G_{r} G_{t} \lambda^{\prime 2} \cdot|h|^{2} \cdot K} \cdot N \cdot\left(Q^{-1}\left(\frac{b}{2 \sin \left(\frac{\pi}{2^{b+1}}\right)} \cdot B E R\right)^{2}\right) P A R_{P S K}
$$

For the transmission of the video data the energy consume by the PA for per bit is defined as followsfor MQAM and MPSK modulation

$$
E_{\text {bit_PA }}=P_{P A} / b \cdot R_{S}
$$

In the PA significant amount of energy is consumed for the transmission and other parameters also affect the energy consumption in RF circuit. Energy consumed by the RF front end can be expressed as

$$
E_{b i t}=\frac{P_{E}+P_{P A}}{b \cdot R_{S}}
$$

Energy consumption for the QAM modulation

$$
\begin{gathered}
E_{b i t}=\frac{P_{E}}{b \cdot R_{s}}+\frac{16 \pi^{2} \cdot d^{2} \cdot L}{3 G_{r} G_{t} \lambda^{\prime 2} \cdot|h|^{2} \cdot K}\left(2^{b}-1\right) \cdot \frac{N}{b \cdot R_{s}} \cdot\left(Q ^ { - 1 } \left(\frac{1}{4}(1-\right.\right. \\
\left.\left.\left.\frac{1}{2^{b / 2}}\right)^{-1} b \cdot B E R\right)^{2}\right) P A R_{Q A M}
\end{gathered}
$$

Energy consumption for the PSK modulation

$E_{b i t}=$
$\frac{P_{E}}{b \cdot R_{S}}+\frac{4 \pi^{2} \cdot d^{2} \cdot L}{3 G_{r} G_{t} \lambda^{\prime 2} \cdot|h|^{2} \cdot K} \cdot \frac{N}{b \cdot R_{S}} \cdot\left(Q^{-1}\left(\frac{b}{2 \sin \left(\frac{\pi}{2^{b+1}}\right)} \cdot B E R\right)^{2}\right) P A R_{P S K}$ 


\section{RESULT ANALYSIS}

We analyses the Doppler Effect on the energy consumption in video transmission over ad hoc network. Figure $2 \& 3$ (a), (b), (c), (d) and (5) represent the energy consumption according to BER for 4QAM, 8QAM, 16QAM, 32QAM, 64QAM respectively. In figure 2 energy consumption and BER performance when average velocity of mobiledevices is $4 \mathrm{~km} / \mathrm{h}$
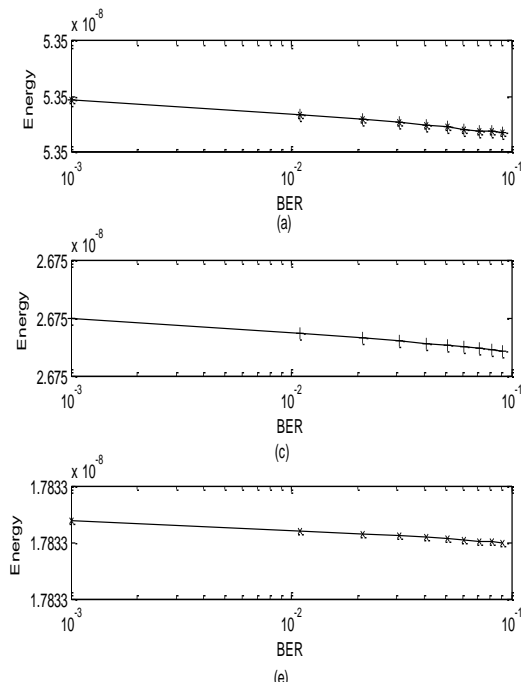

Fig 2: Energy Consumption at average velocity of $4 \mathrm{Km} / \mathrm{h}$ of the mobile nodes
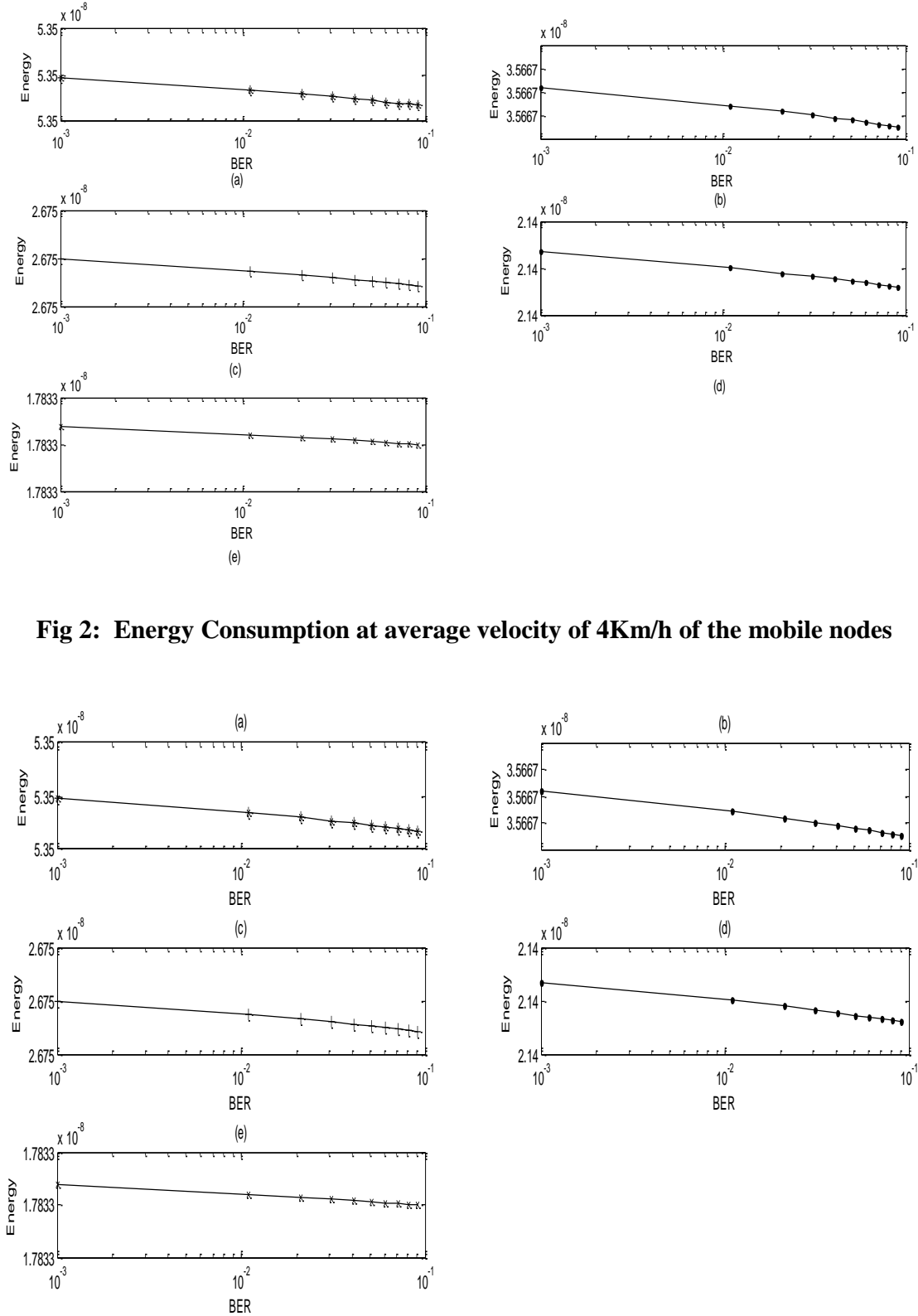

(d) of $40 \mathrm{~km} / \mathrm{h}$ for the mobile nodes.For QAM modulation higher is the modulation level, lower is the energy consumption.The graphs of figure 2 and 3 show that as modulation level the transmission energy consumption decreases. Therefore, for the higher QAM modulation, energy consumption is lower.

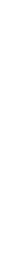


on Communication, Biomedical Engineering and Informatics, 17-19 Oct 2009. BMEI '09, 1-5.

[3] ZHANG, Y., LI, Y., QIAO, D.; 2011. "Energy-Quality System Design for Wireless" Communication. Journal of Networks, North America,. 2011

[4] Wang, R.; Cox, D.; 2002, "Double mobility mitigates fading in ad hoc wireless networks," IEEE International Symposium Antennas and Propagation Society, 2002, vol.2, 306- 309 .

[5] Iranli, A., Choi, K., and Pedram, M., 2003, "Energy-Aware Wireless Video Streaming," in Proc. of the Workshop on Embedded Systems for Real-Time Multimedia, October 2003.

[6] Tianqi Wang; Heinzelman, W.; Seyedi, A.; 2008, "Minimization of transceiver energy consumption in wireless sensor networks with AWGN channels," 46th Annual Allerton Conference on Communication, Control, and Computing,23-26 Sept. 2008,62-66,

[7] Ye Li; Reisslein, M.; Chakrabarti, C.; 2009, "EnergyEfficient Video Transmission Over a Wireless Link,"IEEE Transactions on Vehicular Technology, 2009, vol.58, no. $3,1229-1244$,

[8] ByungJoon Oh; Chang Wen Chen; , "Energy efficient h.264 videotransmission over wireless ad hoc networks based on adaptive 802.11e EDCA MAC protocol," Multimedia and Expo, 2008 IEEE International Conference on, vol., no., pp.1389-1392, June 23 2008April 262008

[9] Ye Li; Bakkaloglu, B.; Chakrabarti, C.; 2007, "A System Level Energy Model and Energy-Quality Evaluation for Integrated Transceiver Front-Ends," IEEE Transactions on Very Large Scale Integration (VLSI) Systems, 2007, vol.15, no.1,90-103,

[10] Kaddar, L.; Hadjadj-Aoul, Y.; Mehaoua, A.; , "EVAN: Energy-Aware SVC Video Streaming over Wireless Ad Hoc Networks," Vehicular Technology Conference (VTC Spring), 2011 IEEE 73rd, vol., no., pp.1-5, 15-18 May 2011

[11] Kihwan Choi, Kwanho Kim, and MassoudPedram. 2003. "Energy-aware MPEG-4 FGS streaming." In Proceedings of the 40th annual Design Automation Conference (DAC '03). ACM, New York, NY, USA, 912-915.

[12] KyungtaeKang, Won J. Jeon, Kyung-Joon Park, Roy H. Campbell, and KlaraNahrstedt. 2010. "Cross-Layer Quality Assessment of Scalable Video Services on Mobile Embedded Systems" IEEE Transactions on Mobile Computing 9, 10 (October 2010), 1478-1490. 